\title{
Treatment of Tongue Lymphangioma with Intralesional Combination Injection of Steroid, Bleomycin and Bevacizumab
}

\author{
Jungil Hwang ${ }^{1}$, \\ Yung Ki Lee', \\ Jin Sik Burm ${ }^{1}$ \\ ${ }^{1}$ Department of Plastic Surgery, Kyung \\ Hee University College of Medicine, Seoul; \\ ${ }^{2}$ Department of Plastic Surgery, Graduate \\ School, Kyung Hee University, Seoul, Korea \\ No potential conflict of interest relevant to \\ this article was reported.
}

\begin{abstract}
Lymphangioma is a congenital malformed lymphatic tumor that rarely involves the tongue. In our clinic, a 10-year-old female presented with lymphangioma circumscriptum involving the right two-thirds of the tongue. We administered an intralesional combination injection of triamcinolone, bleomycin, and bevacizumab as a treatment. Almost complete remission after combination therapy was achieved without complications such as edema, swallowing difficulties or recurrence. Bevacizumab, an inhibitor of vascular endothelial growth factor, was effective for the treatment of lymphangioma of the tongue in this case. No recurrence was noted at the 1-year follow up.
\end{abstract}

Keywords: Lymphangioma / Tongue / Steroid / Bleomycin / Bevacizumab

\section{INTRODUCTION}

Lymphangioma is a congenital benign tumor of malformed lymphatic vessels [1,2]. It is usually located in the head and neck. However, tongue lymphangioma has rarely been reported [3-6], and several such cases introduced surgical treatment or sclerotherapy [3-7]. However, complete excision of tongue lymphangioma is exceedingly difficult, especially for large lesions, owing to diffuse infiltration into tongue structures [5,7]. Vital structural injuries can cause swallowing and speech problems. Sclerotherapy also has certain limitations. The posterior third or extensive involvement of the tongue might require airway protection with tracheostomy or sedation to prevent chewing the swollen tongue during treatment [8]. These patients require intensive care unit (ICU) admis-

\footnotetext{
Correspondence: Jin Sik Burm

Department of Plastic Surgery, Kyung Hee University College of Medicine, 23

Kyungheedae-ro, Dongdaemun-gu, Seoul 02447, Korea

E-mail: jsburm@gmail.com

*This article was presented at the 5th Research and Reconstructive Forum of the Korean Society of Plastic and Reconstructive Surgeons on May 14-16, 2015, in Gangwon Province, Korea
}

Received September 2, 2016 / Revised January 2, 2017 / Accepted January 2, 2017 sion and a prolonged post-injection intubation period until the significant swelling subsides [8]. Intralesional steroid injection has also been used to treat tongue lymphangioma [4]; however, curable treatment is not guaranteed with steroid injection only. Here, we present a case of lymphangioma circumscriptum with extensive tongue involvement that was successfully treated with a staged, combinational, intralesional injection with a minimum dose of steroid, bleomycin, and bevacizumab.

\section{CASE REPORT}

A 10-year-old girl presented with an approximately $70 \times 35 \times 20$ $\mathrm{mm}$ reddish, verrucous, protruded lesion on the tongue (Fig. 1A). The lesion was detected 4 years ago and showed occasional bleeding. Mild swelling first appeared on the right side and gradually progressed to involve the left also. No specific treatment was administered. Examination revealed a yellowish and dark brownish thickening of the tongue. Multiple hemorrhagic, translucent vesicles were observed. Regional lymphadenopathy was absent. On palpation, the swelling was compressible and soft. Head/neck 

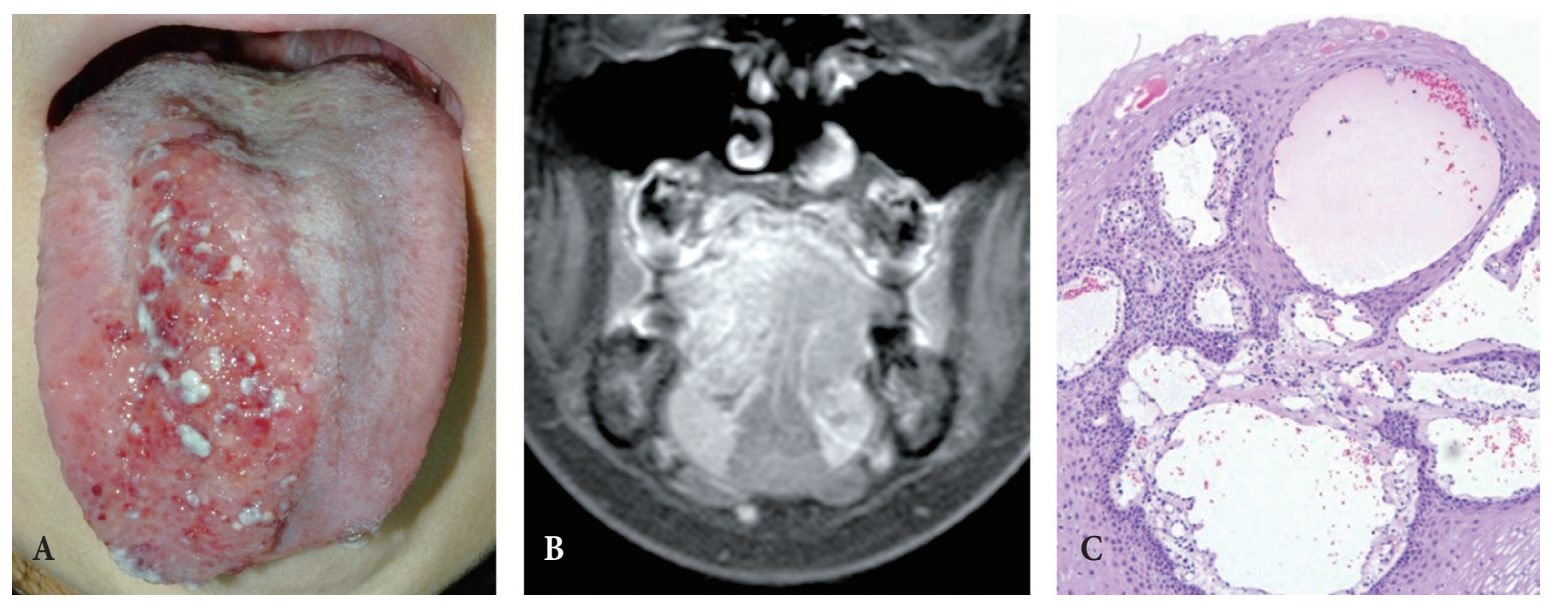

Fig. 1. Pretreatment tongue lymphangioma lesion. (A) An approximately $70 \times 35 \times 20 \mathrm{~mm}$ lesion with multiple reddish vesicles mixed with whitish plaque is observed on the right side of the tongue. (B) Magnetic resonance image shows an approximately $50 \times 35 \times 25 \mathrm{~mm}$ ill-defined lesion in the right side of the tongue, extending to the posterior sublingual space and genioglossus muscle. (C) Histopathological features of lymphangioma circumscriptum reveal a papillary mucosal nodule. Superficially, proliferation of lymphatic vessels is noted $(\mathrm{H} \& \mathrm{E}, \times 100)$.

magnetic resonance imaging revealed a $50 \times 35 \times 25 \mathrm{~mm}$ ill-defined lesion in the right two-thirds of the tongue, posterior sublingual space, and genioglossus (Fig. 1B). Incisional biopsy confirmed lymphangioma circumscriptum (Fig. 1C).

Combination triamcinolone and bleomycin was directly injected in the tongue at monthly intervals. Table 1 shows the detailed injection ratios. Prophylactic intravenous antibiotics were administered before each intralesional injection. The initial triamcinolone concentration was greater than the bleomycin concentration to prevent post-injection swelling and airway obstruction because steroids inhibit the synthesis of multiple inflammatory proteins [9]. After the first treatment, the lymphangioma decreased in size substantially (Fig. 2A). Each month, as the lymphangioma and tongue size decreased, the triamcinolone concentration was reduced and the bleomycin concentration was increased. Eight months after the first treatment, the lesion size stabilized (Fig. 2B). The lymphatic malformation had decreased substantially, but complete remission was not achieved. Intralesional bevacizumab was subsequently included with the triamcinolone/bleomycin combination. The lesion began to regress again. The bevacizumab and bleomycin levels were increased and the steroid concentration was decreased in the monthly injection. Four further intralesional injections were administered. After 1 year of treatment, almost complete regression was achieved, but multiple small vesicles on the tongue surface had not resolved. Tangential excision of the vesicles was performed, resulting in a nearly even tongue surface. Thereafter, a minimum dose of combination injection was administered every

Table 1. Treatment resume table of combinational injection dose of triamcinolone, bleomycin and bevacizumab

\begin{tabular}{lcccc}
$\begin{array}{l}\text { Treatment } \\
\text { sessions }\end{array}$ & Date & $\begin{array}{c}\text { Triamcinolone } \\
(40 \mathrm{mg} / \mathrm{mL})\end{array}$ & $\begin{array}{c}\text { Bleomycin } \\
(1 \mathrm{mg} / \mathrm{mL})\end{array}$ & $\begin{array}{c}\text { Bevacizumab } \\
(25 \mathrm{mg} / \mathrm{mL})\end{array}$ \\
\hline 1st & $2013-01-15$ & 0.6 & 0.2 & 0 \\
\hline 2nd & $2013-01-31$ & 0.5 & 0.3 & 0 \\
\hline 3rd & $2013-02-28$ & 0.4 & 0.4 & 0 \\
\hline 4th & $2013-03-28$ & 0.3 & 0.5 & 0 \\
\hline 5th & $2013-05-02$ & 0.2 & 0.6 & 0 \\
\hline 6th & $2013-06-03$ & 0.2 & 0.8 & 0 \\
\hline 7th & $2013-07-04$ & 0.2 & 1.0 & 0 \\
\hline 8th & $2013-08-06$ & 0.2 & 0.6 & 0.2 \\
\hline 9th & $2013-09-05$ & 0.2 & 0.9 & 0.2 \\
\hline 10th & $2013-10-10$ & 0.1 & 1.0 & 0.3 \\
\hline 11th & $2013-11-04$ & 0.1 & 1.0 & 0.3 \\
\hline 12th & $2013-12-05$ & 0.1 & 0.8 & 0.4 \\
\hline 13th & $2014-02-07$ & 0.1 & 0.4 & 0.2 \\
\hline 14th & $2014-06-23$ & 0.1 & 0.1 & 0.1 \\
\hline 15th & $2014-10-02$ & 0.1 & 0.1 & 0.1 \\
\hline 16th & $2015-01-16$ & 0.1 & 0.1 & 0.1 \\
\hline
\end{tabular}

Values are presented as number $(\mathrm{mL})$. 

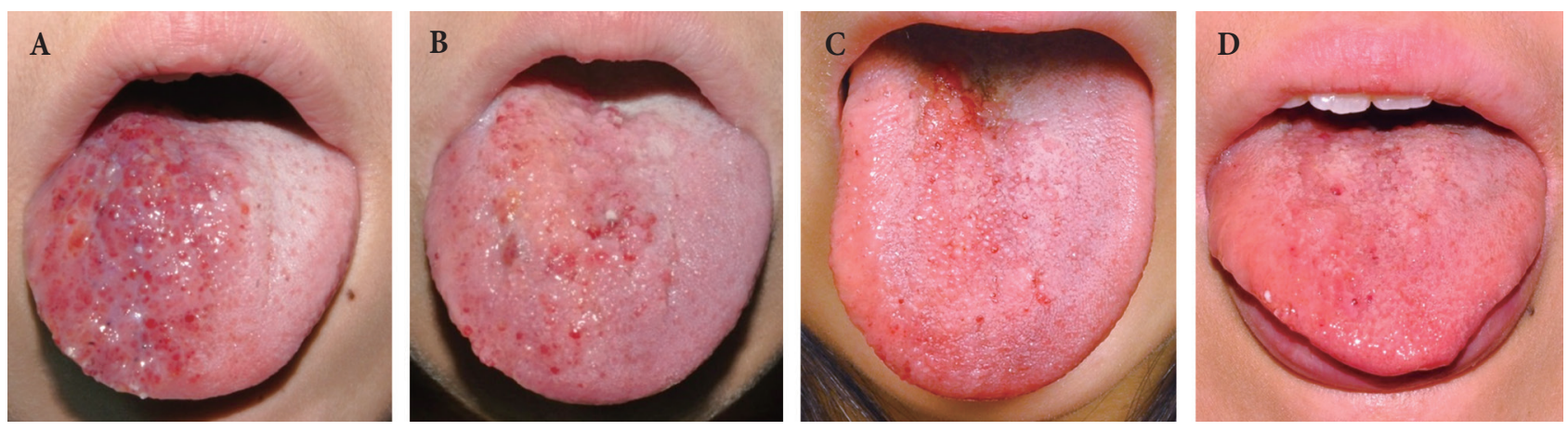

Fig. 2. Post-treatment lesion of tongue lymphangioma. (A) Two weeks after the first treatment. (B) Eight months after the first treatment. (C) Two years after the first treatment. (D) Three years after the first treatment. One year after finishing the treatment.

3 months for another year (Fig. 2C). After finishing the 1-year treatment, 3 years after the initial treatment, neither recurrence nor complications were noted (Fig. 2D).

\section{DISCUSSION}

Lymphangiomas are relative rare, benign, non-encapsulated lesions composed of sequestered, non-communicating lymphoid tissue lined by lymphatic endothelium. They are caused by lymphatic drainage obstruction, abnormal budding of lymphatic tissue, and sequestered lymphatic rests that retain their embryonic growth potential [10]. They are usually diagnosed in infancy and early childhood. Common locations are the head and neck, and tongue involvement is uncommon [2].

Lymphangiomas are classified by lymphatic cavity size. Lymphangioma circumscriptum or capillary lymphangiomas are vesicular masses composed of capillary-sized lymphatic channels. Cavernous lymphangiomas contain larger dilated sinuses. Cystic hygromas are the largest cystic structures. These categories overlap, and the surrounding tissue determines the physical structure of lymphangiomas. Smaller cystic forms tend to occur where the restrictive nature of surrounding tissues limits expansion. Larger forms are found more in areas of loose fatty tissue, where expansion occurs easily [2]. The present case was diagnosed as lymphangioma circumscriptum.

Several possible approaches exist to treat lymphangiomas. Complete tumor excision is the most desirable treatment. Incompletely removed lymphangiomas have a significantly higher re- currence rate than those that are completely removed [2]; however, complete excision of tongue lymphangiomas is exceedingly difficult, especially with large lesions, owing to infiltration into vital structures. Operative complications including airway obstruction, nerve injury, wound infection, and scar formation are also significant. Vital structure injuries can lead to difficulty with swallowing or speech [5]. Operative morbidity and incomplete resection limit surgical excision of diffuse infiltrative tongue lymphangiomas.

Sclerosing agents such as bleomycin, OK-432, tetracycline, cyclophosphamide, and hypertonic saline are also commonly used to treat lymphangiomas $[2,6]$. Literature abounds on the treatment of congenital lymphatic anomalies of the head and neck with bleomycin, which is an antiviral, antibacterial, and antitumor glycopeptide. Its main biochemical action is DNA strand scission with free radical release when the core is oxidized. It also has a sclerosing effect on the vessel endothelium [11]. Previous case reports introduced intralesional bleomycin injection as tongue lymphangioma treatment. However, the posterior third or extensive involvement might require airway protection with tracheostomy or sedation to prevent chewing the swollen tongue during treatment [8]. These patients require ICU admission and post-injection intubation until the mucosal swelling subsides [8]. Therefore, treating large tongue lymphangioma with bleomycin has limitations.

Tongue lymphangioma treatment with intralesional steroids has also been reported. Intralesional steroids cause a significant increase in mast cell density, reduced transcription of cytokines, 
decreased platelet-derived growth factor A and B, and decreased basic fibroblast growth factor. These mechanisms alter cellular functions, resulting in regression of the lymphatic malformation without a significant inflammation reaction $[4,9]$. However, curable treatment is not guaranteed with steroid injection only.

Vascular endothelial growth factor (VEGF) is a serum protein that promotes angiogenesis. VEGFs are significantly increased in proliferative lymphangioma vessels, which are almost absent in normal lymphoendothelial cells $[12,13]$. Steroid therapy increases the mast cell number and cytokine concentration and decreases platelet-derived growth factor and interleukin-6. The therapy contributes to regression of the lymphatic malformation, but does not alter VEGF [9]. Bevacizumab, a recombinant humanized monoclonal antibody originally generated from mice immunized with recombinant human VEGF [14], binds to VEGF and prevents VEGF binding to VEGF receptors [15]. Blockade of VEGF by bevacizumab is used as an effective treatment for capillary hemangioma and diabetic retinopathy $[13,16]$ and has been proved to be a treatment for lymphatic malformation [12,17].

In this case, lymphangioma involved the tongue base and posterior tongue, which hindered surgical excision and required airway protection during sclerotherapy. Thus, we initially planned to treat the lymphangioma with a combination of steroid and bleomycin. Triamcinolone prevented the inflammation, edematous changes, and airway obstruction that can occur after injection of high-dose bleomycin alone. Both the steroid and bleomycin helped reduce the lymphangioma. Combinational therapy made it possible to decrease the bleomycin dose to only $0.1-1.2 \mathrm{mg} / \mathrm{ses}-$ sion, which was substantially lower than the conventional doses (1-10 mg/session) [8]. With lower doses of bleomycin, the lymphangioma regressed substantially without post-injection swelling and airway obstruction, but complete regression was not achieved with steroid and bleomycin only.

We reviewed articles about the characteristics of lymphatic malformation and decided to administer bevacizumab also. Intralesional injection of bevacizumab for tongue lymphangioma has not been reported yet. After adding bevacizumab to the triamcinolone/bleomycin combination, the lesion regressed again and near-complete remission was achieved. The timing here is a bit unclear. Therefore, I suggest the following replacement: after 1 year of treatment with the triamcinolone/bleomycin combination, a minimum dose of the combined bevacizumab/triamcinolone/bleomycin injection was administered every 3-months for 1 more year. After finishing the 1-year treatment, no increase in the lesion size was noted.

The present case showed satisfactory regression after injection of intravenous antibiotics followed by intralesional injection of a steroid/bleomycin/bevacizumab combination. No morbidity was noted, and intubation was not required. Combination therapy enabled a low dose of each treatment, which reduced the systemic and local side effects caused by single therapy. Combination injection maximized the synergy effect during treatment. We think that the initial effective volume ratio of triamcinolone $(40 \mathrm{mg} /$ $\mathrm{mL})$, bleomycin $(1 \mathrm{mg} / \mathrm{mL})$, and bevacizumab $(25 \mathrm{mg} / \mathrm{mL})$ might be 1:2:1 or 1:3:1. Intralesional combination therapy could be a treatment option for tongue lymphangioma with extensive involvement.

\section{REFERENCES}

1. Paletta FX. ymphangioma. Plast Reconstr Surg 1966;37:269-79.

2. Brock ME, Smith RJ, Parey SE, Mobley DL. Lymphangioma. An otolaryngologic perspective. Int J Pediatr Otorhinolaryngol 1987;14:13340.

3. Iamaroon A, Pongsiriwet S, Srisuwan S, Krisanaprakornkit S. Lymphangioma of the tongue. Int J Paediatr Dent 2003;13:62-3.

4. Khurana S, Vij A, Singal A, Pandhi D. Lymphangioma of the tongue: response to intralesional steroids. J Dermatolog Treat 2006;17:124-6.

5. Kataria P, Passey JC, Agarwal AK. Lymphangioma circumscriptum of the tongue: successful treatment using intralesional bleomycin. J Laryngol Otol 2009;123:1390-2.

6. Chakravarti A, Bhargava R. Lymphangioma circumscriptum of the tongue in children: successful treatment using intralesional bleomycin. Int J Pediatr Otorhinolaryngol 2013;77:1367-9.

7. Saijo M, Munro IR, Mancer K. Lymphangioma: a long-term followup study. Plast Reconstr Surg 1975;56:642-51.

8. Sainsbury DC, Kessell G, Fall AJ, Hampton FJ, Guhan A, Muir T. Intralesional bleomycin injection treatment for vascular birthmarks: a 5-year experience at a single United Kingdom unit. Plast Reconstr Surg 2011;127:2031-44.

9. Hasan Q, Tan ST, Gush J, Peters SG, Davis PF. Steroid therapy of a proliferating hemangioma: histochemical and molecular changes. Pediatrics 2000;105:117-20. 
10. Mosca RC, Pereira GA, Mantesso A. Cystic hygroma: characterization by computerized tomography. Oral Surg Oral Med Oral Pathol Oral Radiol Endod 2008;105:e65-9.

11. Gooding C, Meyer D. Intralesional bleomycin: a potential treatment for refractory orbital lymphangiomas. Ophthal Plast Reconstr Surg 2014;30:e65-7.

12. Aman J, Thunnissen E, Paul MA, van Nieuw Amerongen GP, VonkNoordegraaf A. Successful treatment of diffuse pulmonary lymphangiomatosis with bevacizumab. Ann Intern Med 2012;156:839-40.

13. Steeples LR, Bonshek R, Morgan L. Intralesional bevacizumab for cutaneous capillary haemangioma associated with pregnancy. Clin Exp Ophthalmol 2013;41:413-4.

14. Presta LG, Chen H, O’Connor SJ, Chisholm V, Meng YG, Krummen L, et al. Humanization of an anti-vascular endothelial growth factor monoclonal antibody for the therapy of solid tumors and other disorders. Cancer Res 1997;57:4593-9.

15. Ellis LM. Mechanisms of action of bevacizumab as a component of therapy for metastatic colorectal cancer. Semin Oncol 2006;33:S1-7.

16. Spaide RF, Fisher YL. Intravitreal bevacizumab (Avastin) treatment of proliferative diabetic retinopathy complicated by vitreous hemorrhage. Retina 2006;26:275-8.

17. Grunewald TG, Damke L, Maschan M, Petrova U, Surianinova O, Esipenko A, et al. First report of effective and feasible treatment of multifocal lymphangiomatosis (Gorham-Stout) with bevacizumab in a child. Ann Oncol 2010;21:1733-4. 